\title{
Expression of pulmonary aquaporin 1 is dramatically upregulated in mice with pulmonary fibrosis induced by bleomycin
}

\author{
Xiwen Gao', Guifang Wang'2, Wei Zhang', Qing Peng', Min Xue ${ }^{1}$, Hu Jinhong ${ }^{3}$
}

1Department of Pulmonary Medicine, Minhang District Central Hospital, Ruijin Hospital Group, Shanghai, PR China

2Department of Pulmonary Medicine, Zhongshan Hospital, Fudan University, Shanghai, PR China

3Department of Pharmacy, Changhai Hospital, Second Military Medical University, Shanghai, PR China

Submitted: 7 August 2011

Accepted: 13 February 2012

Arch Med Sci 2013; 9, 5: 916-921

DOI: $10.5114 /$ aoms.2012.31011

Copyright @ 2013 Termedia \& Banach

\section{Abstract}

Introduction: Pulmonary fibrosis occurs due to fibroblast proliferation and collagen production in the lung and begins with alveolar inflammatory edema. Aquaporins (AQPs) play pivotal roles in lung fluid transport. In this study we establish the experimental model for pulmonary fibrosis in C57BL/6 mice to investigate expressions of AQP1 and AQP5 in lung tissue.

Material and methods: Mice in model groups were treated intratracheally with bleomycin with the dose of $5 \mathrm{mg} / \mathrm{kg}$ body weight. The mice were sacrificed at 1 week, 2 weeks and 3 weeks respectively. The left upper lungs were harvested for histopathologic H-E and Masson's staining. The mRNAs of AQP1 and AQP5 were analyzed by real-time polymerase chain reaction (real-time PCR) and the proteins of AQP1 and AQP5 were analyzed by western blotting.

Results: Real-time PCR showed that AQP1 mRNA in bleomycin $1 \mathrm{w}, 2 \mathrm{w}$, and $3 \mathrm{w}$ groups increased by $377 \%, 880 \%$ and $823 \%$ respectively compared to that in the control group $(p<0.01)$. Western blotting showed that the expression of AQP1 protein in bleomycin $1 \mathrm{w}, 2 \mathrm{w}$, and $3 \mathrm{w}$ groups increased by $53 \%, 144 \%$, and $141 \%$, respectively $(p<0.05)$. AQP5 mRNA in bleomycin $1 w$ and $2 w$ group decreased by $78 \%$ and $66 \%$, respectively $(p<0.05)$. In bleomycin $2 w$ and $3 w$ groups it decreased by $69 \%$ and $80 \%(p<0.05)$.

Conclusions: The expression of AQP1 dramatically increased in pulmonary fibrosis. AQP1 plays an important role in the progress of pulmonary fibrosis.

Key words: aquaporin 1, aquaporin 5, pulmonary fibrosis, bleomycin.

\section{Introduction}

Aquaporin (AQP) is a cell-membrane protein that exists widely in plants, animals, and bacteria. In 1984, the AQP system was separated from bovine lens proteins, as the membrane-integral protein 26 [1]. Until now 13 kinds of AQP (AQP0 to AQP12) have been described, collectively referred to as $A Q P s[2]$. The water that is necessary for cell metabolism is transported by AQPs, which have a highly selective ability to aid water to cross biological membranes [3]. In recent years, more and more attention has been paid to the protein expression profile of the aquaporin (AQP) family mem-

\section{Corresponding author:} Prof. Hu Jinhong Department of Pharmacy Changhai Hospital Second Military Medical University No. 168, Changhai Road 200433 Shanghai, PR China Phone: 86-21-81873747 Fax: +86 21-81873747

E-mail: hujinhong2008@sina.com 
bers in acute lung injury and lung cancer $[4,5]$. To different extents, it has been reported that AQP1 and AQP5 were correlated with lung injury [6], and decreased expression of AQP5 in bleomycin-induced lung fibrosis was found [7]. But AQP1 in pulmonary fibrosis has not been investigated. AQP1 is expressed in the apical and basolateral membrane of the microvascular endothelium, as well as in the visceral pleura [8].

Pulmonary fibrosis is a parenchymal lung disease characterized by progressive interstitial fibrosis [9]. Predicting risk factors remain unknown though lung fibroblasts maybe in terms of proinflammatory mediators and pro-fibrotic pathways. The persistence of alveolitis causes progressive disruption and destruction of the alveolar architecture, which can result in end-stage fibrosis [10]. A well-characterized animal model of human lung fibrosis is induced by bleomycin. Administration of bleomycin in animals results in an acute pan-alveolitis with pulmonary edema in the first week, followed by a 2 to 3 week subacute phase characterized by proliferation of fibroblasts and synthesis of extracellular matrix proteins $[11,12]$. It is necessary for the resolution of acute lung injury to remove the pulmonary edema fluid from the alveolar space. This will probably prevent its late fibrotic consequences. The importance of the resolution of alveolitis has recently been shown by the fact that the removal of alveolar edema fluid early is associated with a better clinical outcome, specifically with a lower mortality rate in lung injury $[13,14]$. We can find new therapeutic targets for pulmonary fibrosis resulting from lung injury by evaluating changes in the expression of aquaporins involved in the clearance of pulmonary edema.

Pulmonary inflammation and edema in mice are associated with a marked reduction in the expression levels of AQP1 and AQP5 in the distal lung, suggesting that the AQP1 and AQP5 in abnormal fluid fluxes have a role during pulmonary inflammation [15]. In the present study, we assessed the expression of AQP1 and AQP5 in lung tissue with pulmonary fibrosis induced by bleomycin. It showed that AQP1 dramatic up-regulation was positively related to the severity of pulmonary fibrosis. Furthermore, AQP5 down-regulation is presented at some time. A better understanding of the expression of AQP1 in pulmonary fibrosis could be critical in finding new treatments for this life-threatening disease.

\section{Material and methods}

\section{Animals}

Male eight-week-old C57BL/6 mice purchased from Fudan University Animal Breeding and Research Center (Shanghai, China) were housed and cared for using protocols approved by Research Ani- mal Care of Shanghai Changhai Hospital. Mice were treated intratracheally with bleomycin (Nippon Kayaku Kabushiki Kaisha, Japan) with a dose of $5 \mathrm{mg} / \mathrm{kg}$ body weight on day 0 . The mice were sacrificed at 1 week, 2 weeks and 3 weeks. The left upper lungs were harvested for histopathological $\mathrm{H}-\mathrm{E}$ and Masson's staining. Forty-eight mice were divided into 4 groups: a bleomycin $1 \mathrm{w}$ group, a bleomycin 2 w group, a bleomycin $3 \mathrm{w}$ group and a control group treated with the same volume of normal saline.

\section{Histopathological evaluation}

The left upper lung tissues dehydrated in graded ethanol were embedded in paraffin, for sectioning into $4 \mu \mathrm{m}$ and staining with hematoxylin and eosin $(H \& E)$. The slides were reviewed by 3 pathologists. The overall severity of fibrosis was determined by the pattern and intensity of interstitial inflammation according to the Szapiel 1979 working classification scale [16].

\section{RT-PCR of AQP1 and AQP5}

The samples homogenized with the appropriate volume of TRIzol (Invitrogen, USA) solution were placed in centrifuge tubes for mRNA extraction and RT-PCR. The AQP1 upstream primer sequence was 5'CTGGCCTTTGGTTTGAGCAT3' and the downstream primer sequence was 5'CCACACACTGGGCGATGAT3', a total of 150 base pairs (bp). The AQP5 upstream primer sequence was 5'GCGCTCAGCAACACAAC3' and the downstream primer sequence was 5' GTGTGACCGACAAGCCAATG3', a total of $150 \mathrm{bp}$. The $\beta$-actin upstream primer sequence was 5'ACGGCCAGGTCATCACTATTG3' and the downstream primer sequence was 5'TGGATGCCACAGGATTCCAT3'. The 150 bp PCR products from each sample including AQP1, AQP5 and $\beta$-actin were analyzed on $1 \%$ agarose gels (Promega, USA). The final results visualized on an ultraviolet light box were photographed on Kodak film. The density of each band was compared with a 150-bp band that expressed $\beta$-actin. The intensity ratio of the difference was later used to compare the results after fibrosis.

\section{Immunohistochemical analysis of AQP1 and AQP5}

Anti-AQP1 specificity antibody (AB3065) and antiAQP5 specificity antibody (AB3069) were purchased from Chemicon International Group (Canada). The sections were washed with phosphate buffered saline (PBS) ( $\mathrm{pH}$ 7.4) thrice for 3 min each time. The sections were fixed with $3 \% \mathrm{H}_{2} \mathrm{O}_{2}$ for 15 min at room temperature, and washed with PBS thrice, each time for $5 \mathrm{~min}$. After PBS washing 3 more times, we added anti-AQP1 or anti-AQP5 antibody at $4^{\circ} \mathrm{C}$ for $12 \mathrm{~h}$, and washed with PBS thrice further. 
Table I. Semi-quantitative scores of pulmonary fibrosis $(\bar{x} \pm s)$

\begin{tabular}{|lcc|}
\hline Group & $n$ & Scores \\
\hline Control & 12 & $1.00 \pm 0.00$ \\
\hline Bleomycin $1 \mathrm{w}$ & 11 & $1.60 \pm 0.89^{*}$ \\
\hline Bleomycin $2 \mathrm{w}$ & 10 & $2.00 \pm 0.71^{*}$ \\
\hline Bleomycin $3 \mathrm{w}$ & 8 & $3.60 \pm 0.55^{* \#}$ \\
\hline${ }^{*} p<0.05$ vs. control group, $\# p<0.05$ vs. bleomycin 1 w group
\end{tabular}

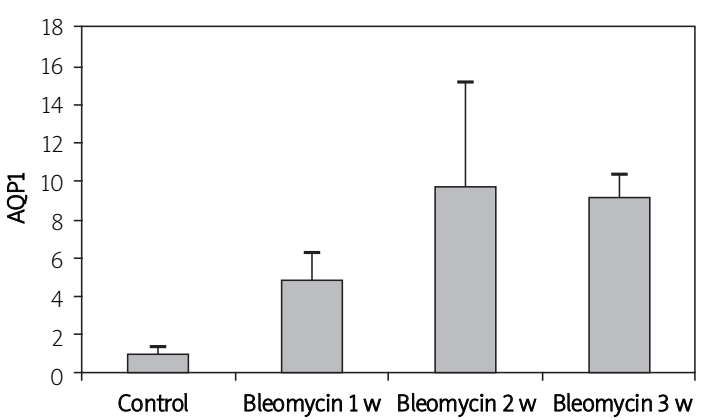

Figure 1. Determine AQP1 mRNA expression by real time PCR in mice with pulmonary fibrosis induced by bleomycin

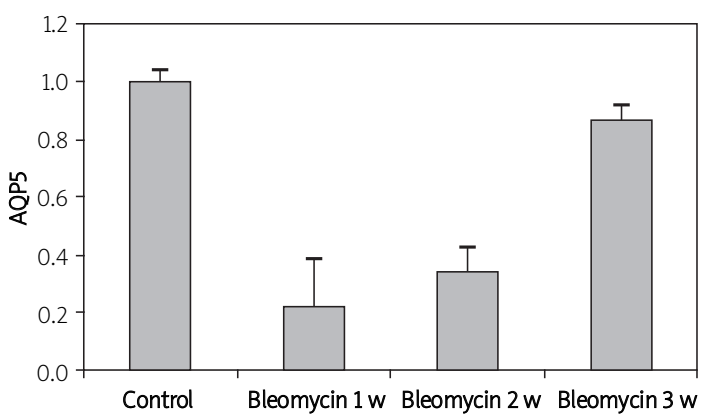

Figure 2. Determine AQP5 mRNA expression by real time PCR in mice with pulmonary fibrosis induced by bleomycin

Then the second antibody was added and the section maintained at $37^{\circ} \mathrm{C}$ for $12 \mathrm{~h}$, washed with PBS 3 more times and counterstained. The sections were then examined under a microscope. The epithelial cell membranes IN Collection system which has a brown staining WAS positive cells. Image analysis of the gray value of positive cells (IOD values) yielded the relative expression of AQP1 and AQP5 proteins.

\section{Statistical analysis}

Statistical analysis was performed using SPSS 11.0 for Windows and Student's $t$-test and ANOVA for repeated measures considering values of $p<0.05$ to be significant.

\section{Results}

\section{Histopathological expression}

After 1 week, the mice were treated with bleomycin, we observed the fact that some mice died. There was one in the $1 \mathrm{w}$ group, two in the $2 \mathrm{w}$ group and four in the $3 \mathrm{w}$ group. The bleomycin groups showed pulmonary fibrosis at days 7, 14 and 21 and displayed higher scores compared with normal controls $(p<0.05)$. The PF score increased gradually week by week. The PF score was higher in the $3 \mathrm{w}$ group compared with the $1 \mathrm{w}$ group $(p<0.05$, Table I).

\section{AQP1 and AQP5 mRNA Expression}

To evaluate serial changes in AQP1 and AQP5 associated with pulmonary fibrosis, RT-PCR was used to analyze mRNA expression at days 7, 14 and 21 after the mice were treated with bleomycin. Real-time PCR showed that AQP1 mRNA expression in bleomycin $1 \mathrm{~W}$, $2 \mathrm{w}$, and $3 \mathrm{w}$ groups increased by $3.77,8.8$ and 8.23 times respectively compared to that in the control group ( $p<0.05$ ) (Figure 1). AQP5 mRNA expression in the bleomycin $1 \mathrm{w}$ and $2 \mathrm{w}$ group decreased by $78 \%$ and $66 \%$ respectively $(p<0.05)$, while there was no significant difference between the bleomycin $3 \mathrm{w}$ group and the control group $(p>0.05)$ (Figure 2).

\section{$\mathrm{AQP1}$ and $\mathrm{AQP5}$ protein expression}

Immunohistochemistry results showed that AQP1 was expressed on microvascular endothelial cells. Staining of AQP1 showed increased immunoreactivity in bleomycin groups compared to that in the control group, especially in the severe fibrotic areas (Figure 3), while AQP5 was expressed on type I pneumocytes in the alveolus. Staining of AQP5 showed decreased immunoreactivity in bleomycin groups compared to that in the control group (Figure 4).

Western blotting showed that AQP1 protein expression in bleomycin groups was significantly upregulated at days 7, 14 and 21 compared with the normal control group $(p<0.05)$. Expression of AQP1 protein in bleomycin $1 \mathrm{w}, 2 \mathrm{w}$, and $3 \mathrm{w}$ groups increased by $53 \%, 144 \%$, and $141 \%$ respectively of the control group value. There were no differences in AQP1 protein expression among the bleomycin groups (Table II, Figure 5). AQP5 protein expression in the bleomycin $1 \mathrm{w}$ group decreased by $13 \%$ compared to that in the control group $(p>0.05)$ and in the bleomycin $2 \mathrm{w}$ and $3 \mathrm{w}$ groups it decreased by $69 \%$ and $80 \%$ respectively $(p<0.05)$ (Table III, Figure 6).

\section{Discussion}

The pathogenesis of pulmonary fibrosis is not clear but it appears to be the consequence of acute, persistent, or recurrent lung injury and inflammation [17]. Following this inflammatory response, increased vascular permeability and pulmonary 

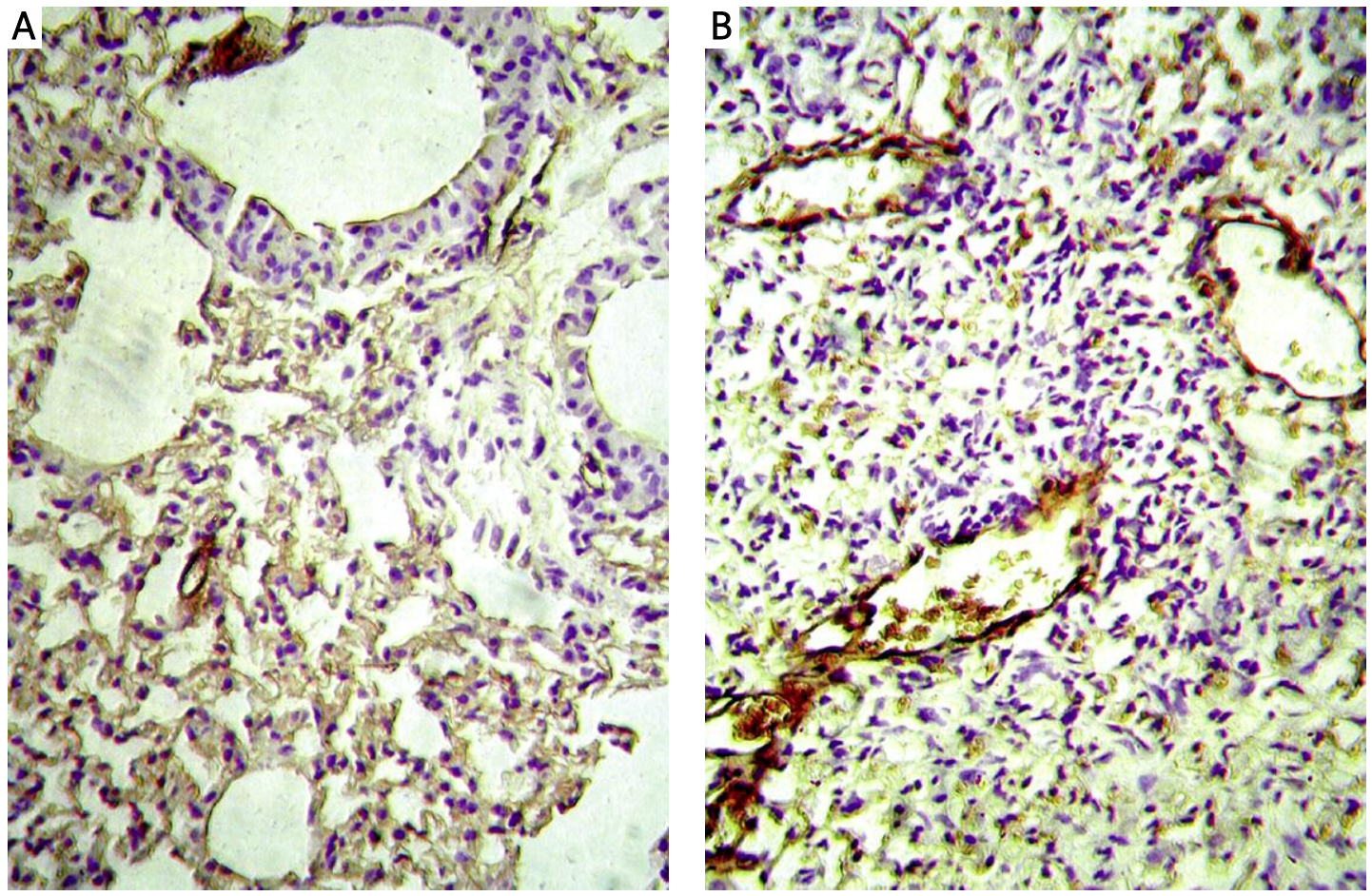

Figure 3. AQP1 protein expression is showed in immunohistochemistry. A - Control group, B - bleomycin $3 \mathrm{w}$ group
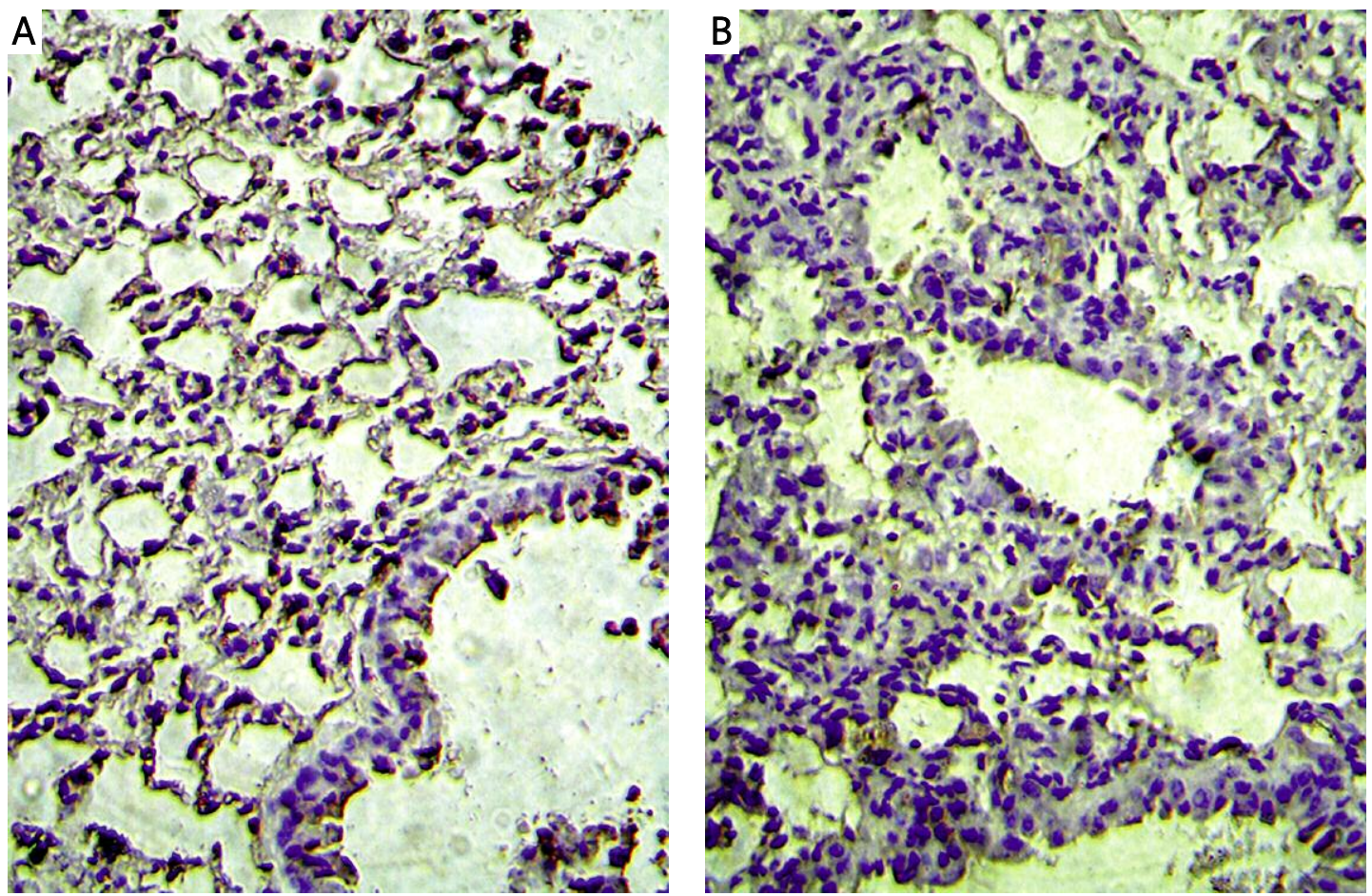

Figure 4. AQP5 protein expression is showed in immunohistochemistry. A-Control group, B - bleomycin $3 \mathrm{w}$ group

edema ensue [18]. The pathway for the flow of water across the endothelial and epithelial lung barrier is not completely elucidated, but recently AQPs have been described as playing a critical role in water removal from the lung extracellular space
[19]. Decreased expression of AQP1 and AQP5 has also been described in pathological conditions of the lung, such as acute lung injury [8]. However, alteration of AQP1 and AQP5 expression in chronic inflammatory processes such as that observed in 
Table II. AQP1 protein expression determined by Western blotting $(\bar{x} \pm s)$

\begin{tabular}{|llc|}
\hline Group & $n$ & Gray scale ratio \\
\hline Control & 8 & $0.34 \pm 0.05$ \\
\hline Bleomycin 1 w & 8 & $0.52 \pm 0.14^{\star}$ \\
\hline Bleomycin 2 w & 6 & $0.83 \pm 0.17^{\star}$ \\
\hline Bleomycin 3 w & 6 & $0.82 \pm 0.11^{\star}$ \\
\hline
\end{tabular}

${ }^{*} p<0.05$ vs. control group. Gray scale ratio - AQP1 protein/GAPHD

Table III. AQP5 protein expression determined by Western blotting $(\bar{x} \pm s)$

\begin{tabular}{|lcc|}
\hline Group & $n$ & Gray scale ratio \\
\hline Control & 8 & $3.32 \pm 0.80$ \\
\hline Bleomycin 1 w & 8 & $2.88 \pm 0.72$ \\
\hline Bleomycin 2 w & 6 & $1.04 \pm 0.25^{\star}$ \\
\hline Bleomycin 3 w & 6 & $0.65 \pm 0.21^{\star}$ \\
\hline
\end{tabular}

${ }^{*} p<0.05$ vs. control group. Gray scale ratio - AQP5 protein/GAPHD

lung fibrosis has not as yet been reported. In the present study, we evaluated the protein and RNA expression of AQP1 and AQP5 in a mouse model of bleomycin-induced lung fibrosis. Both the protein and mRNA expression of AQP1 were significantly increased in the fibrotic tissue of our animal models, suggesting that insufficient AQP1-mediated transportation of extracellular liquid may also take place in lung fibrosis. However, at the present time we cannot be sure that the increase of AQP1 expression is secondary to the fibrosis. Either way, it is clear that AQP1 may be a useful marker for pulmonary injury owing to fibrosis in the mouse.

In our fibrosis model, the expression of AQP1 was increased in microvascular endothelial cells. The mechanism of this AQP1 increase is unclear. Previous studies have shown that the majority of osmotically driven water transport in lung microvascular endothelial cells occurs by a transcellular route through AQP1 water channels. AQP1 facilitates hydrostatically driven lung edema, but is not required for active near-isomolar absorption of alveolar fluid [20]. In contrast to a previous study [15], AQP1 expression was increased in the lungs of rats at 7, 14 and 21 days after bleomycin treatment. This suggests that AQPS may be differentially expressed according to tissue injury type and that epithelial cells' response to injury differ. Further studies are needed to evaluate the mechanism involved in the regulation of fluid movement and inflammation. Towne et al. [15] suggested that decreases in both AQP1 and AQP5 may contribute to edema by reducing the transcellular rate of excess water removal, thereby effectively trapping water in the alveolar and interstitial spaces. Although AQP5 expression was decreased at some time in our study, AQP1 expression was

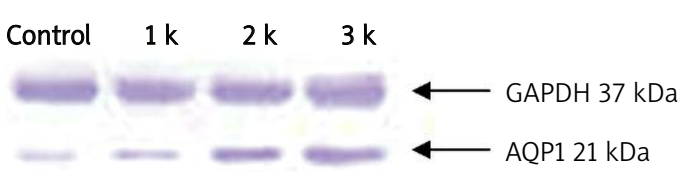

Figure 5. Determine AQP1 protein expression by Western-blotting

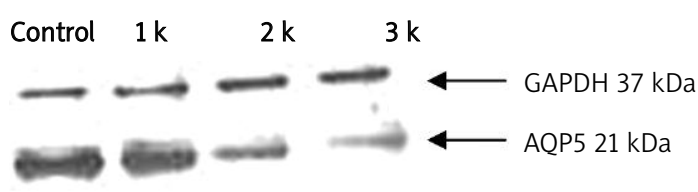

Figure 6. Determine AQP5 protein expression by Western-blotting

increased dramatically. AQP1 may be more important in edema development following bleomycin inhalation, and the inability of pulmonary vascular endothelial cells to clear the air space may be an important factor in the inflammatory process of pulmonary fibrosis. Increased expression of AQP-1 has been reported in the frontal cortex of patients with Creutzfeldt-Jacob disease [21] and on the pleura of rats with a tuberculous pleural effusion [22]. The different types of injury and the different time points of the same injury might cause different alterations in AQP-1.

It was found by Gabazza et al. [7] that the expression of AQP5 protein was significantly decreased in pulmonary edema resulting from bleomycin treatment. But in our study the decrease was less at 21 days, indicating that the return of AQP5 expression to baseline levels was coincident with a lessening of the inflammatory response. Previous studies [23] have shown that treatment of murine lung epithelial cells with tumor necrosis factor- $\alpha$ (TNF- $\alpha$ ) results in a dependent decrease of concentration and time in AQP5 mRNA and protein expression, suggesting that TNF- $\alpha$ may play an important role in AQP5 down-regulation in the inflamed lung. Interestingly, the loss of AQP5 in epithelial cells was not only observed in the fibrotic lung of mice that had received intratracheal instillation of bleomycin but also in those receiving continuous infusion of bleomycin through a subcutaneous mini-pump, suggesting that both acute and chronic lung injuries induce reduction in AQP5 expression. In our study AQP5 mRNA expression started to return but protein expression decreased further. It showed that pulmonary fibrosis impaired the cellular membrane not damaged to genetic transcription.

The biological significance of abnormal extracellular accumulation of fluid in the lung from patients with lung fibrosis is unclear. Pulmonary edema may play an active role in the development of lung fibrosis. Impairment of clearance of edema in patients with acute lung injury and interstitial 
lung disease is associated with poor clinical outcome [24]. Some previous studies have shown the development of lung fibrosis with the presence of chronic pulmonary edema after lung injury $[25,26]$. In our model of lung fibrosis, we found a dramatic increase in the expression of AQP1. The chronic lung injury induced by bleomycin was probably the result of injected bleomycin entering into the systemic circulation at slow pace.

In conclusion, the results of the present study show that chronic lung injury and lung fibrosis is associated with up-regulated expression of AQP1 in the lung. Further studies will be necessary to clarify the cause-effect relationship between AQP1 up-regulation and the development of pulmonary fibrosis.

\section{References}

1. Dibas Al, Mia AJ, Yorio T. Aquaporins (water channels): role in vasopressin-activated water transport. Proc Soc Exp Biol Med 1998; 219: 183-99.

2. Itoh T, Rai T, Kuwahara M, et al. Identification of a novel aquaporin, AQP12, expressed in pancreatic acinar cells. Biochem Biophys Res Commun 2005; 330: 832-8.

3. Walz T, Fujiyoshi Y, Engel A. The AQP structure and functional implications. Handb Exp Pharmacol 2009; 190: 31-56.

4. Su X, Song Y, Jiang J, Bai C. The role of aquaporin-1 (AQP1) expression in a murine model of lipopolysaccharideinduced acute lung injury. Respir Physiol Neurobiol 2004; 142: 1-11.

5. Hoque MO, Soria JC, Woo J, et al. Aquaporin 1 is overexpressed in lung cancer and stimulates NIH-3T3 cell proliferation and anchorage-independent growth. Am J Pathol 2006; 168: 1345-53.

6. Towne JE, Harrod KS, Krane CM, Menon AG. Decreased expression of aquaporin (AQP) 1 and AQP5 in mouse lung after acute viral infection. Am J Respir Cell Mol Biol 2000; 22: 34-44.

7. Gabazza EC, Kasper M, Ohta K, et al. Decreased expression of aquaporin-5 in bleomycin-induced lung fibrosis in the mouse. Pathol Int 2004; 54: 774-80.

8. King LS, Nielsen S, Agre P. Aquaporin-1 water channel protein in lung: ontogeny, steroid-induced expression, and distribution in rat. J Clin Invest 1996; 97: 2183-91.

9. Barlo NP, van Moorsel CH, van den Bosch JM, Grutters JC. Predicting prognosis in idiopathic pulmonary fibrosis. Sarcoidosis Vasc Diffuse Lung Dis 2010; 27: 85-95.

10. Tobin MJ. Tuberculosis, lung infections, interstitial lung disease, and journalology in AJRCCM 2002. Am J Respir Crit Care Med 2003; 167: 345-55.

11. Yasui H, Gabazza EC, Tamaki S, et al. Intratracheal administration of activated protein C inhibits bleomycin-induced lung fibrosis in the mouse. Am J Respir Crit Care Med 2001; 163: 1660-8.

12. Jones HA, Schofield JB, Krausz T, Boobis AR, Haslett C. Pulmonary fibrosis correlates with duration of tissue neutrophil activation. Am J Respir Crit Care Med 1998; 158: 620-8.

13. Ware LB, Matthay MA. Alveolar fluid clearance is impaired in the majority of patients with acute lung injury and the acute respiratory distress syndrome. Am J Respir Crit Care Med 2001; 163: 1376-83.

14. Matthay MA. Alveolar fluid clearance in patients with ARDS: does it make a difference? Chest 2002; 122 (6 Suppl): 340S-3S.

15. Towne JE, Harrod KS, Krane CM, Menon AG. Decreased expression of aquaporin (AQP) 1 and AQP5 in mouse lung after acute viral infection. Am J Respir Cell Mol Biol 2000; 22: 34-44.

16. Szapiel SV, Elson NA, Fulmer JD, Hunninghake GW, Crystal RG. Bleomycin-induced interstitial pulmonary disease in the nude, athymic mouse. Am Rev Respir Dis 1979; 120: 893-9.

17. Ward PA, Hunninghake GW. Lung inflammation and fibrosis. Am J Respir Crit Care Med 1998; 157: S123-9.

18. Chakinala M, Schuster DP. Pulmonary edema. In: Fishman AP, Elias JA, Fishman JA, Grippi MA, Kaiser LR, Senior RM (eds.). Fishman's pulmonary diseases and disorders. 3rd ed.: McGraw-Hill, New York 2002; 469-75.

19. King LS, Agre P. Man is not a rodent: aquaporins in the airways. Am J Respir Cell Mol Biol 2001; 24: 221-3.

20. Bai C, Fukuda N, Song Y, Ma T, Matthay MA, Verkman AS. Lung fluid transport in aquaporin-1 and aquaporin-4 knockout mice. J Clin Invest 1999; 103: 555-61.

21. Rodríguez A, Pérez-Gracia E, Espinosa JC, Pumarola M, Torres JM, Ferrer I. Increased expression of water channel aquaporin 1 and aquaporin 4 in Creutzfeldt-Jakob disease and in bovine spongiform encephalopathy-infected bovine-PrP transgenic mice. Acta Neuropathol 2006; 112: 573-85.

22. Du H, Xie C, He Q, Deng X. Increased expression of aquaporin-1 on the pleura of rats with a tuberculous pleural effusion. Lung 2007; 185: 325-36.

23. Towne JE, Krane CM, Bachurski CJ, Menon AG. Tumor necrosis factor-alpha inhibits aquaporin 5 expression in mouse lung epithelial cells. J Biol Chem 2001; 276: 18657-64.

24. Blivet S, Philit F, Sab JM, et al. Outcome of patients with idiopathic pulmonary fibrosis admitted to the ICU for respiratory failure. Chest 2001; 120: 209-12.

25. Koike K, Chida M, Suzuki S, et al. Pulmonary fibrosis after endotoxin-induced permeability edema. Nihon Kyobu Shikkan Gakkai Zasshi 1989; 27: 440-4.

26. Selman M, King TE, Pardo A. Idiopathic pulmonary fibrosis: prevailing and evolving hypotheses about its pathogenesis and implications for therapy. Ann Intern Med 2001; 134: 136-51. 